\title{
The Cross-Correlation Relationship Between the Real Estate Industry and High-Quality Economic Development
}

\author{
Mengwan ZHANG* \\ School of Economics and Management, Beijing University of Technology, Beijing 100124, China \\ E-mail: alicezmw@163.com \\ Fengfeng GAO \\ School of Economics and Management, Beijing University of Technology, Beijing 100124, China \\ E-mail: gao_flora@163.com \\ Jialin GAO \\ School of Economics and Management, Tsinghua University, Beijing 100084, China \\ E-mail: betterjl@163.com \\ Weiguo CHEN \\ College of Economics and Management, Beijing University of Technology, Beijing 100124, China \\ E-mail: chungweiguo@126.com
}

Chong Aik LEE

UCSI University, Selangor 56000, Malaysia

E-mail: chongaiklee@icloud.com

\begin{abstract}
Based on China's real estate investment (REI), commercial housing sales (CHS), and GDP data from 2001 to 2020, we studied the dependence, asymmetry, and transmission direction of China's real estate and economy. Their cross-correlation is demonstrated through the deep crosscorrelation analysis (DCCA) algorithm, multifractal asymmetric detrended cross-correlation analysis (MF-ADCCA) algorithm and DCCA algorithm based on a time delay. Both REI and CHS have persistence with GDP. The real estate industry and economy have asymmetric multifractal characteristics. Meanwhile, real estate and the economy have different trends, and they can affect each other with the same length of time delay. This effect diminishes in a short period of time, and there are unsteady fluctuations in cross-correlation persistence in the long term.
\end{abstract}

Keywords real estate industry; high-quality economic development; cross-correlation relationship

\section{Introduction}

High-quality economic development refers to a cumulative result and is based on the quantity of economic growth. The cumulative result includes an efficiency improvement in the

Received June 10, 2021, accepted October 19, 2021

Supported by Postdoctoral Research Foundation of Chaoyang District (Q1011019202101), Beijing Postdoctoral Research Foundation (Q6011019202101), Special Project for Soft Science Research of Hebei Provincial Science and Technology Plan (215576107D)

*Corresponding author 
economic growth process, stability improvement, structural optimization, improved innovation capacity and welfare distribution ${ }^{[1]}$. Real estate industry development has taken a major position in economic development. However, an over-reliance on real estate investment (REI) can also destabilize the economy. For instance, housing bubble foam can influence the industrial structure and resource allocation efficiency and maintain stable economic development ${ }^{[2]}$. Both real estate development and high-quality economic development have attracted the attention of researchers, and they have been mentioned in the path of China's economic growth ${ }^{[3]}$. In recent years, the real estate industry has been the pillar industry in the national economy. Currently, China is on the way to achieving high-quality economic development, and is real estate industry development able to increase high-quality economic development? The relationship between real estate development and high-quality economic development is very important. This paper will use ten years of monthly data from 2010.02 to 2020.12 to identify the relationship between real estate industry development and high-quality economic development.

\section{Literature Review}

Real estate development has always played essential roles in China's economic development. Recently, scholars have also sought to research the relationship between them. Below, a literature review is conducted on the index selection of real estate development, the methods within research that focus on real estate development and economic development, and the correlation relationship between real estate development and economic development.

1) Index selection for real estate and economic development

According to Liu ${ }^{[4]}$, REI and commercial housing sales (CHS) represent indexes for real estate industry development; GDP represents economic development. Additionally, based on Zhang \& Wang ${ }^{[5]}$, GDP represents economic development. Bhowmik et al. ${ }^{[6]}$ used GDP to represent economic development and compared two other indexes from the stock market to identify the economic status. Wolfgang et al. ${ }^{[7]}$ analysed the real estate industry's economic sector and its recent research dynamics by using machine learning algorithms. Using a statespace model, Tommaso et al. ${ }^{[8]}$ analysed the bubble dynamics in China's real estate market. In research, CHS, GDP, and other indexes have been used as variables to evaluate the data. Balemi, et al. ${ }^{[9]}$ reviewed the impact of COVID-19 on the real estate industry market and cited papers from the USA, Italy, China, etc. Future research should consider the deeper possible relevance of financial and real estate development and the economic market.

2) Empirical methods

In terms of the empirical methodology, Liu, et al. ${ }^{[10]}$ used the Mankiw-Romer-Weil (MRW) model, selected 2000-2006 Chinese economic time series data, revealed the mechanism of the effect of real estate development on economic growth, and provided theoretical support for further real estate policy development. Based on an econometric model, Zhou, et al. ${ }^{[11]}$ performed an empirical analysis. The results proved an inverted U-shaped relationship between REI and the quality of economic growth. In other words, to ensure the quality of the economy, it is necessary to optimize REI. Jing \& Wang ${ }^{[12]}$ analysed 2000-2016 panel data on the effects of REI on economic growth. Using the Markov zone system transfer model, Zhang ${ }^{[13]}$ conducted research on real estate development in Beijing city and stated the risk of REI. Fan, et al. ${ }^{[14]}$ used 
the time varying parameter-stochastic volatility-vector autoregression (TVP-VAR) model to discover the correlation relationship among REI, CHS, innovation, and manufacturing industry investment.

3) The relevance between real estate development and economic development

Most scholars agree that REI could be helpful for economic growth. Based on an econometric method, $\mathrm{Wu} \& \mathrm{Lu}^{[15]}$ tested the REI effect on economic development. The results show that a change in REI is the opposite of a change in economic development. Therefore, regulating the real estate market in China is necessary. Otherwise, REI will not be able to push economic development. Zhang \& Teng ${ }^{[16]}$ proved the hypothesis that there is an inverted U-shaped relationship between CHS and the economy. This means that with real estate development, economic development will have a downward trend. Although existing studies have calculated the effects of REI on GDP, excessive REI is not conducive to economic development. An early real estate development period, real estate development, and CHS can drive economic increases when the sale price exceeds a reasonable interval; real estate development can restrict economic development ${ }^{[17,18]}$.

Some scholars consider real estate development to be unnecessary to increase economic development. For example, based on 286 panel data points from 2004 to 2015, Li \& Wang ${ }^{[19]}$ identified that real estate development can affect high-quality economic growth and that there is an inverted U-shaped relationship between them. Chen, et al. ${ }^{[20]}$ stated that stimulating the real estate market may promote economic development, but it may also restrain economic development. The real estate industry does not sustainably affect economic growth, and the economic growth rate will increase or decrease, depending on different situations ${ }^{[21]}$. In addition, Zhou, et al. ${ }^{[11]}$ stated that the current research area focuses on real estate development and economic development rather than high-quality economic development. On the other hand, REI and high-quality economic development need more theory and empirical research to support the present period because high-quality economic development is currently a requirement in China ${ }^{[22]}$.

Compared to existing research, this paper uses the deep cross-correlation analysis (DCCA) algorithm, multifractal asymmetric detrended cross-correlation analysis (MF-ADCCA) algorithm and DCCA algorithm based on a time delay to prove the results. It pays attention to the dependency, asymmetry, and conduction direction relationship between real estate development and high-quality economic development. Therefore, this paper contributes to promoting the collaborative development between the real estate industry and high-quality economic development. Meanwhile, this paper complements research on real estate development and high-quality economic development.

\section{Methodology}

\subsection{Dependency Study — DCCA Algorithm}

The DCCA algorithm focuses on the identity of the cross-correlation relationship of two time series. It is an expansion of the detrended fluctuation analysis (DFA) algorithm based on the autocorrelation of one time series. Five steps will be presented as follows.

Step 1 Set up two identical time series $\left\{x^{(1)}(k)\right\}$ and $\left\{x^{(2)}(k)\right\}$, where $k=1,2, \cdots, N$ 
and $N$ represents the amount of data within the time series; thus, set up a new time series:

$$
y^{(i)}(m)=\sum_{k=1}^{m}\left(x^{(i)}(k)-\overline{x^{(i)}}\right), \quad m=1,2, \cdots, N .
$$

Among them, $\overline{x^{(i)}}=\frac{1}{N} \sum_{k=1}^{N} x^{(i)}(k), i=1,2$.

Step 2 New time series $y^{(i)}(m)=\sum_{k=1}^{m}\left(x^{(i)}(t)-\overline{x^{(i)}}\right)(i=1,2)$ can be divided into nonoverlapping subintervals with a length of $n$, and the number of subintervals is $N_{n}=\operatorname{int}(N / n)$. Since $N$ cannot be aliquoted by $n$, to include the sequence in the end, the above operation is repeated to settle the data in reverse order. Therefore, the same length subinterval is obtained, and the length of the subinterval is $2 N_{n}$.

Step 3 For the number of $\mathrm{n}$ data points, every subinterval $j\left(j=1,2, \cdots, 2 N_{n}\right)$ can be fitted through the least squares method. The fitting function can be expressed as $L_{Y_{j}^{(i)}}$, where $j=1,2, \cdots, 2 N_{n}$ and $i=1,2$. Thus, the correlation coefficient formula can be expressed as follows:

While $j=1,2, \cdots, N_{n}$,

$$
F^{2}(n, j)=\frac{1}{n} \sum_{t=1}^{n}\left|y^{(1)}((j-1) n+t)-L_{Y_{j}^{(1)}}\right| \cdot\left|y^{(2)}((j-1) n+t)-L_{Y_{j}^{(2)}}\right| .
$$

While $j=N_{n}+1, N_{n}+2, \cdots, 2 N_{n}$,

$$
F^{2}(n, j)=\frac{1}{n} \sum_{t=1}^{n}\left|y^{(1)}\left(N-\left(j-N_{n}\right) n+t\right)-L_{Y_{j}^{(1)}}\right| \cdot\left|y^{(2)}\left(N-\left(j-N_{n}\right) n+t\right)-L_{Y_{j}^{(2)}}\right|,
$$

where $t=1,2 \cdots n$.

Step 4 Determine the trend cross wave function.

$$
F^{2}(n)=\left[\frac{1}{2 N_{n}} \sum_{j=1}^{2 N_{n}} F(n, j)^{\frac{1}{2}}\right] .
$$

Step 5 On the condition that there is a long-term cross relevance during sequences $\left\{x^{(1)}(k)\right\}$ and $\left\{x^{(2)}(k)\right\}$, the relationship between function $F^{2}(n)$ and time distance $n$ has a low-power relationship:

$$
F^{2}(n) \sim n^{h(2)}
$$

The above formula can be expressed as follows:

$$
\ln \left(F^{2}(n)\right)=h(2) \ln (n)+\ln C,
$$

where $C$ is a constant number and $h(2)$ is called the exponent of the scale and can be expressed through a $\log$-log coordinate area's hill fitting curve to composition with $\ln \left(F^{2}(n)\right)$ and $\ln (n)$. If both initial sequences are the same, the DCCA method is equivalent to the DFA method.

Repeat Steps 2 4 for different $n$, and $F^{2}(n)$ will increase following the increase in $n$, which means that $h(2)$ always has a positive situation. When $h(2)>0.5$, the cross-relevance relationship during two sequences is continuous with a positive situation. This means that if one 
sequence has a rising trend, the other sequence will have a higher possibility of a rising trend. When $0<h(2)<0.5$, the cross-relevance relationship during the two sequences is persistently negative. When $h(2)=0.5$, there is no long-range cross-correlation during the two sequences. This means that a change in one sequence does not cause a change in the other sequence.

\subsection{Asymmetric Study - MF-ADCCA Algorithm}

The MF-ADCCA algorithm aims to identify the asymmetric cross-correlation relationship between real estate and high-quality economic development. This means that it studies the cross-correlation with different directions of change between real estate and high-quality economic development and the relationship between different ranges of change. Since Step 1 and Step 2 of the MF-ADCCA algorithm are the same as those of the DCCA algorithm, the next step will be presented as follows:

Step 3 The order sub-time series is $S_{j}^{(i)}=\left\{s_{j, k}^{(i)}, k=1,2, \cdots, n\right\}$, and the consolidation time series of the corresponding subintervals is $Y_{j, k}^{(i)}=\left\{y_{j, k}^{(i)}, k=1,2, \cdots, n\right\}$, where $j=1,2, \cdots, 2 N_{n}$. Thus, in the $j$ subintervals,

$$
\begin{aligned}
& s_{j, k}^{(i)}=x^{(i)}((j-1) n+k), \quad y_{j, k}^{(i)}=y^{(i)}((j-1) n+k), \quad j=1,2, \cdots, N_{n}, \\
& s_{j, k}^{(i)}=x^{(i)}\left(\left(j-N_{n}\right) n+k\right), \quad y_{j, k}^{(i)}=y^{(i)}\left(\left(j-N_{n}\right) n+k\right), \quad j=N_{n}+1, \cdots, 2 N_{n} .
\end{aligned}
$$

Step 4 Calculate the local fitting function by the least squares method for time series $S_{j}^{(i)}=\left\{s_{j, k}^{(i)}, k=1,2, \cdots, n\right\}$ and its congruent time series $Y_{j, k}^{(i)}=\left\{y_{j, k}^{(i)}, k=1,2, \cdots, n\right\}$ :

$$
\begin{aligned}
& L_{S_{j}(i)}(k)=a_{S_{j}}^{(i)}+b_{S_{j}}^{(i)} k, \\
& L_{Y_{j}(i)}(k)=a_{Y_{j}}^{(i)}+b_{Y_{j}}^{(i)} k,
\end{aligned}
$$

where $k$ stands for the abscissa, $j=1,2, \cdots, 2 N_{n}$; therefore, the wave function is defined as follows:

$$
F_{j}(n)=\frac{1}{n} \sum_{k=1}^{n}\left|y_{j, k}^{(1)}-L_{Y_{j}^{(1)}}(k)\right| \cdot\left|y_{j, k}^{(2)}-L_{Y_{j}^{(2)}}(k)\right| .
$$

Step 5 The slope $b_{S_{j}}^{i}$ can determine the concentrated sub-time series $x^{(i)}$ such that the near trend of $S_{j}^{(i)}$ becomes positive or negative. If $b_{S_{j}^{(i)}}>0$ (or $b_{S_{j}^{(i)}}<0$ ), it means concentrating sub-time series $x^{(i)}$, and $S_{j}^{(i)}$ is a positive trend (negative trend). Therefore, it can be considered that different trends of certain series can lead to asymmetric cross-correlation during two time sequences for instance, $\left\{x^{(1)}(k)\right\}$. The directivity $q$ average wave function can be expressed as follows:

$$
\begin{aligned}
& F_{q}^{+}(n)=\left(\frac{1}{M^{+}} \sum_{j=1}^{2 N_{n}} \frac{\operatorname{sign}\left(b_{S_{j}^{(1)}}\right)+1}{2}\left[F_{j}(n)\right]^{q / 2}\right)^{1 / q}, \\
& F_{q}^{-}(n)=\left(\frac{1}{M^{-}} \sum_{j=1}^{2 N_{n}} \frac{-\left[\operatorname{sign}\left(b_{S_{j}^{(1)}}\right)-1\right]}{2}\left[F_{j}(n)\right]^{q / 2}\right)^{1 / q},
\end{aligned}
$$


where $M^{+}=\sum_{j=1}^{2 N_{n}} \frac{\operatorname{sign}\left(b_{S_{j}(1)}\right)+1}{2}$ and $M^{-}=\sum_{j=1}^{2 N_{n}} \frac{-\left[\operatorname{sign}\left(b_{S_{j}^{(1)}}\right)-1\right]}{2}$ represent the numbers of subtime sequences with relatively active and inactive trends. On the condition that power law cross-relevance exists, the scaling or power law concern should meet the following:

$$
\begin{aligned}
& F_{q}^{+}(n) \sim n^{H_{12}^{+}(q)}, \\
& F_{q}^{-}(n) \sim n^{H_{12}^{-}(q)},
\end{aligned}
$$

where $H_{12}^{+}(q)$ stands for the ascending scale index of time series $\left\{x^{(1)}(t)\right\}$ and $H_{12}^{-}(q)$ stands for the descending scale index of time sequence $\left\{x^{(1)}(t)\right\}$. While $H_{12}^{+}(q)>0.5$, the increase in the cross-correlation of time sequence $\left\{x^{(1)}(t)\right\}$ is persistent. While $H_{12}^{+}(q)<0.5$, the increase in the cross-correlation of time sequence $\left\{x^{(1)}(t)\right\}$ is anti-persistent. If the change in $H_{12}^{+}(q)$ follows the change in $q$, it means that the cross-correlation between the two sequences has a multifractal feature. Otherwise, there is a single fractal feature.

$H_{12}^{-}(q)$ is the same as $H_{12}^{+}(q)$. On the side, for the purpose of measuring the level of asymmetry in cross-correlation, the following is defined:

$$
\Delta H_{12}(q)=H_{12}^{+}(q)-H_{12}^{-}(q) .
$$

For every data point $q$, the larger $\left|\Delta H_{12}(q)\right|$ is, the stronger the asymmetry. If $\Delta H_{12}(q)>0$, then as the time series $\left\{x^{(1)}(t)\right\}$ rises, the cross-correlation is more persistent. If $\Delta H_{12}(q)<0$, then as time series $\left\{x^{(1)}(t)\right\}$ decreases, the cross-correlation is more persistent. If $\Delta H_{12}(q)=0$, the cross-correlation is symmetrical regardless of the different trends of time series $\left\{x^{(1)}(t)\right\}$. Additionally, for both the rising trend and the decreasing trend of time series $\left\{x^{(1)}(t)\right\}$, their cross-correlations are the same.

\subsection{Conduction Direction Study - DCCA Algorithm Based on a Time Delay}

Regarding time sequences $\left\{x^{(1)}(t)\right\}$ and $\left\{x^{(2)}(t)\right\}$, their DCCA algorithm based on a time delay will be presented below:

Step 1 Order $\left\{x^{(2)}(t)\right\}$ lag $\Delta T$, and set up a new time sequence:

$$
\begin{aligned}
& y^{(1)}(m)=\sum_{t=1}^{m}\left(x^{(1)}(t)-\overline{x^{(1)}}\right), \quad m=1,2, \cdots, N-\Delta T, \\
& y^{(2)}(m)=\sum_{t=1}^{m}\left(x^{(2)}(t+\Delta T)-\overline{x^{(2)}}\right), \quad m=1,2, \cdots, N-\Delta T,
\end{aligned}
$$

where $\overline{x^{(1)}}=\frac{1}{N-\Delta T} \sum_{t=1}^{N-\Delta T} x^{(1)}(t), \overline{x^{(2)}}=\frac{1}{N-\Delta T} \sum_{t=1}^{N-\Delta T} x^{(2)}(t+\Delta T)$, and $\Delta T$ will select any real value that is less than $N$.

Steps $2 \sim 5$ are the same as those of the DCCA algorithm.

\section{Data Sources and Processing}

This paper aims to research the cross-relevance of real estate development and high-quality economic development in China. Referring to $\mathrm{Liu}^{[4]}$, real estate development can be measured based on three indexes: Real estate prices, REI, and CHS. This paper selected two indexes: REI and CHS. For economic development, GDP was selected as the index. Therefore, ten 
years of monthly data were selected from 2010.02 to 2020.12. All data were sourced from the China Statistical Yearbook of the National Bureau of Statistics. Since January data were not calculated in the China Statistical Yearbook, a total of 121 data points for each index were analysed and applied in this paper.

\subsection{Analysis Process of the DCCA Algorithm}

Referring to Hasan \& Salim ${ }^{[23]}, 10<n<N / 4$. Through matrix \& laboratory software (Matlab), Figure 1 and Figure 2 were drawn.

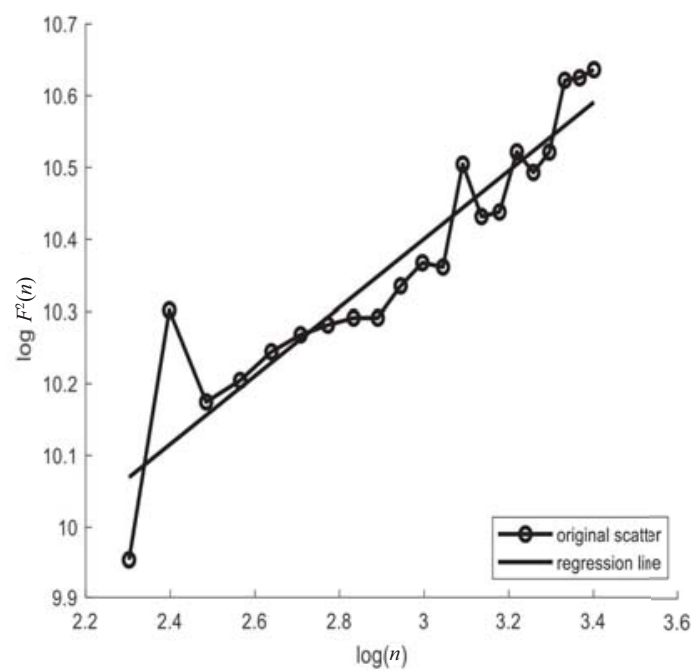

Figure 1 Cross-relevance of F1 and GDP

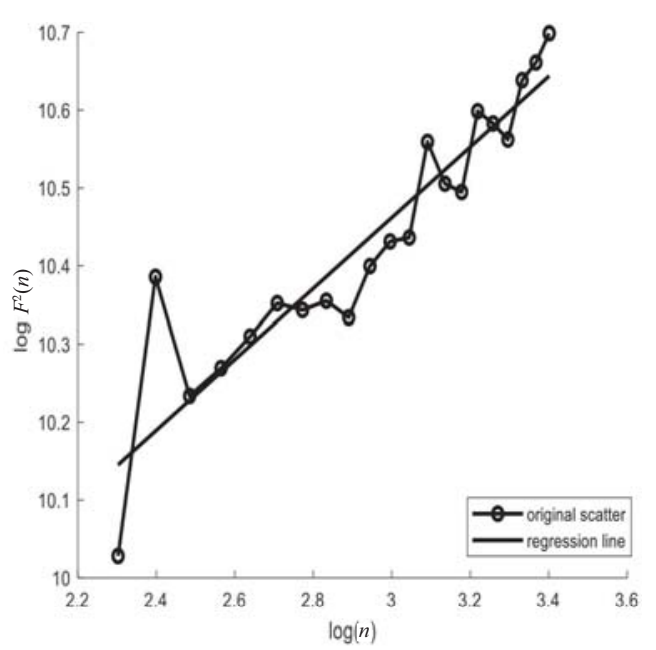

Figure 2 Cross-relevance of F2 and GDP

Figure 1 and Figure 2 show that the cross-correlation of REI and CHS and the GDP slope of the wave equations is 0.4747 and 0.4540 , respectively. The results show that both REI and CHS have persistence with GDP. They also mean that while GDP rises in the current period, it will decrease in the next period if there are no external factors.

\subsection{Analysis Process of the MF-ADCCA algorithm}

In Subsection 3.1, through the analysis process of the DCCA algorithm, the cross-correlation between economic development and real estate development is shown to have persistence. However, the cross-correlation scale index was still in the weak period. Therefore, deep analysis is necessary for subsequent research. Hence, the MF-ADCCA algorithm was used to perform a deep analysis.

First, we analysed the asymmetric cross-correlation of cross sequences with the different trends. Let $q=2$ and obtain the results for $h_{12}^{+}(q=2)$ and $h_{12}^{-}(q=2)$; they represent GDP and REI. Detailed data are presented in Table $1 . h_{12}^{+}(q=2), h_{12}^{-}(q=2)$, and $\Delta h_{12}(q=2)$ represent different direction trends of the growth rate from GDP and REI. According to Table 1 , while the GDP growth rate is on the rise, the value of $h_{12}^{+}(q=2)$ is 0.3333 , which is less than 0.5. While the GDP growth rate shows a declining trend, the value of $h_{12}^{-}(q=2)$ is 0.2408 , which is less than 0.5 . This result means that there is an anti-persistent relationship during cross-relevance. 
Table 1 Index of cross-correlation (GDP and REI)

\begin{tabular}{cccc}
\hline Time sequence & $h_{12}^{+}(q=2)$ & $h_{12}^{-}(q=2)$ & $\Delta h_{12}(q=2)$ \\
\hline GDP & 0.3333 & 0.2408 & 0.0925 \\
REI & 0.3134 & 0.2106 & 0.1028 \\
\hline
\end{tabular}

While the REI growth rate is on the rise, the value of $h_{12}^{+}(q=2)$ is 0.3134 , which is less than 0.5. While the REI growth rate shows a declining trend, the value of $h_{12}^{-}(q=2)$ is 0.2106 , which is less than 0.5. This result means that there is an anti-persistent relationship during cross-relevance. In addition, the value of $\Delta h_{12}(q=2)$ is more than 0 , which means that an asymmetric relationship exists in the cross-correlation between GDP and REI.

Regarding the GDP index and REI index, their $h_{12}^{+}(q=2)$ is more than $h_{12}^{-}(q=2)$, which indicates that while the GDP exponent shows a declining trend, its anti-persistence is stronger than that when the GDP exponent is on the rise. Regarding the REI growth rate, $\Delta h_{12}(q=2)>0$, which means that $h_{12}^{+}(q=2)>h_{12}^{-}(q=2)$. In other words, while the REI growth rate is on the rise, their cross-relevance is situated in a more persistent environment. In addition, while REI has different trends, $\left|\Delta h_{12}(q=2)\right|$ is large, and while the GDP exponent has different directions, $\left|\Delta h_{12}(q=2)\right|$ is small. However, while the GDP index has different trends, a more persistent asymmetric cross-correlation will occur.

Table 2 Trend analysis of crossover sequences (GDP and CHS)

\begin{tabular}{cccc}
\hline Time sequence & $h_{12}^{+}(q=2)$ & $h_{12}^{-}(q=2)$ & $\Delta h_{12}(q=2)$ \\
\hline GDP & 0.2787 & 0.1597 & 0.119 \\
CHS & 0.2741 & 0.1508 & 0.1233 \\
\hline
\end{tabular}

Analysis of the asymmetric cross-correlation of cross-sequences with different trends between GDP and CHS. Let $q=2$ and obtain the results for $h_{12}^{+}(q=2)$ and $h_{12}^{-}(2)$; they represent GDP and CHS. Detailed data are presented in Table 2. $h_{12}^{+}(q=2), h_{12}^{-}(q=2)$, and $\Delta h_{12}(q=2)$ represent different trends of the growth rate of GDP and CHS. According to Table 2, while the GDP growth rate is on the rise, the value of $h_{12}^{+}(q=2)$ is 0.2787 , which is less than 0.5 , while the GDP growth rate shows a declining trend. The value of $h_{12}^{-}(q=2)$ is 0.1597 , which is less than 0.5. This result means that there is an anti-persistent relationship during cross-relevance.

While the CHS growth rate is on the rise, the value of $h_{12}^{+}(q=2)$ is 0.274 , which is less than 0.5. While the REI growth rate shows a declining trend, the value of $h_{12}^{-}(2)$ is 0.1508 , which is less than 0.5 . This result means that there is an anti-persistent relationship during cross-relevance. In addition, the value of $\Delta h_{12}(q=2)$ is more than 0 , which means that an asymmetric relationship occurs in the cross-relevance between GDP and CHS.

Regarding the GDP index and CHS index, their $h_{12}^{+}(q=2)$ is more than $h_{12}^{-}(q=2)$, which indicates that while the GDP exponent shows a declining trend, its anti-persistence is stronger than that when the GDP exponent is on the rise. Regarding the CHS index, $\Delta h_{12}(q=2)>0$, which means that $h_{12}^{+}(q=2)>h_{12}^{-}(q=2)$. In other words, while the CHS growth rate is on the rise, their cross-relevance is more persistent. In addition, while CHS have different tendencies, $\left|\Delta h_{12}(q=2)\right|$ is large, and while the GDP index has different tendencies, $\left|\Delta h_{12}(q=2)\right|$ is 
small. However, while the GDP index has different trends, a more persistent asymmetric crosscorrelation will occur.

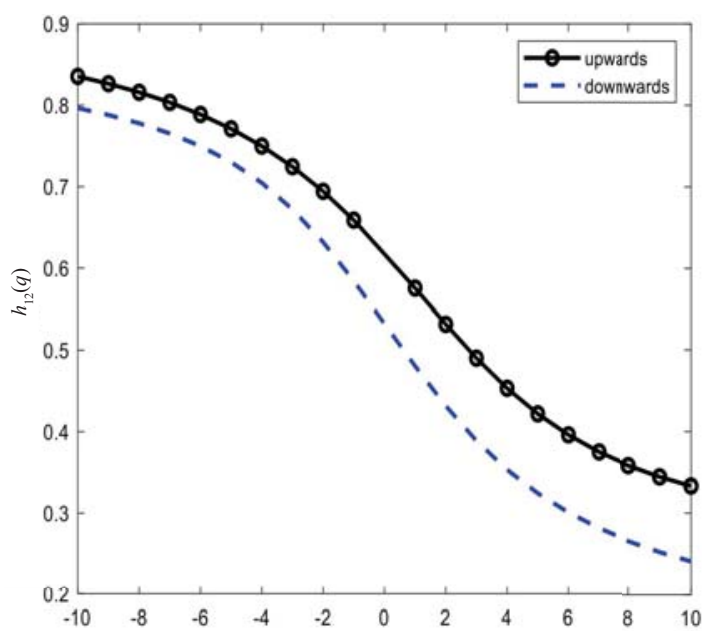

(a) GDP index

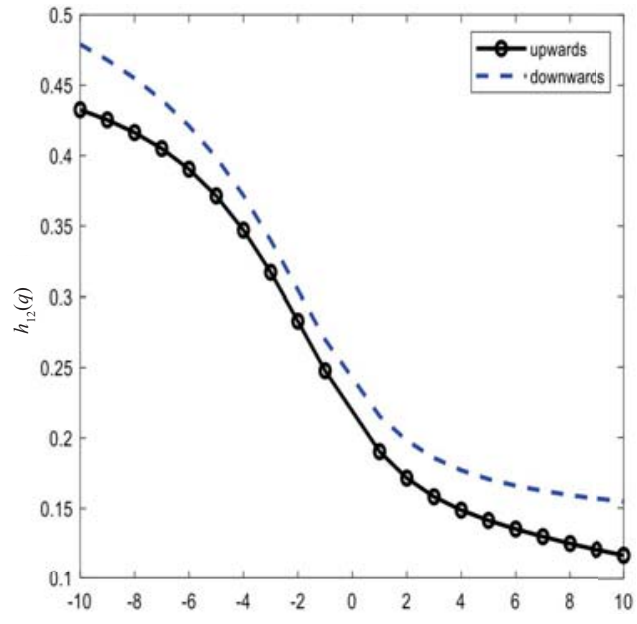

(b) REI index

*Upwards means that the GDP and REI growth rates show a rising trend; downwards means that the GDP and REI growth rates show a declining trend.

Figure 3 Relevance plots for $h_{12}^{+}(q)$ and $h_{12}^{-}(q)$

Second, we analyse the relevance relationship of different trends and the magnitude of fluctuations. For the GDP index, $h_{12}^{+}(q)$ is more than 0.5 for any $q$ value from -10 to $2 . h_{12}^{+}(q)$ is less than 0.5 for any $q$ value from 2 to $10 . h_{12}^{-}(q)$ is more than 0.5 for any $q$ value from -10 to 0 , and $h_{12}^{-}(q)$ is less than 0.5 for any $q$ value from 0 to 10 . This means that both $h_{12}^{+}(q)$ and $h_{12}^{-}(q)$ are not always larger than 0.5 . The effects on the REI growth rate rely on the magnitude of the fluctuations based on the GDP exponent. While the GDP exponent shows a rising trend, the cross-correlation of large fluctuations is less persistent, whereas small fluctuations are more persistent.

Regarding the REI index, both $h_{12}^{+}(q)$ and $h_{12}^{-}(q)$ are less than 0.5 for any $q$ value from -10 to 10. This means that the influence of the REI exponent on the GDP growth rate does not depend on each other, and their relevance is less persistent, while the REI exponent presents a rising trend. In addition, the GDP exponent and REI exponent both have multifractal asymmetric relevance relationships.

Based on Figure 4, both the GDP and REI indexes increase the growth rate with an increase in the value of $q$, and they intersect when $q=4$. Additionally, the direction of the trend indicates that the cross-relevance of REI is slightly stronger than that of GDP.

Regarding the GDP exponent, $h_{12}^{+}(q)$ is more than 0.5 for any $q$ value from -10 to $2, h_{12}^{+}(q)$ is less than 0.5 for any $q$ value from 2 to $10, h_{12}^{-}(q)$ is more than 0.5 for any $q$ value from -10 to 0 , and $h_{12}^{-}(q)$ is less than 0.5 for any $q$ value from 0 to 10 . This means that both $h_{12}^{+}(q)$ and $h_{12}^{-}(q)$ are not always larger than 0.5 . The effects on the CHS growth rate rely on the magnitude of fluctuations based on the GDP exponent. While the GDP exponent is on the rise, the cross-correlation of large fluctuations is less persistent, whereas small fluctuations are more persistent. 


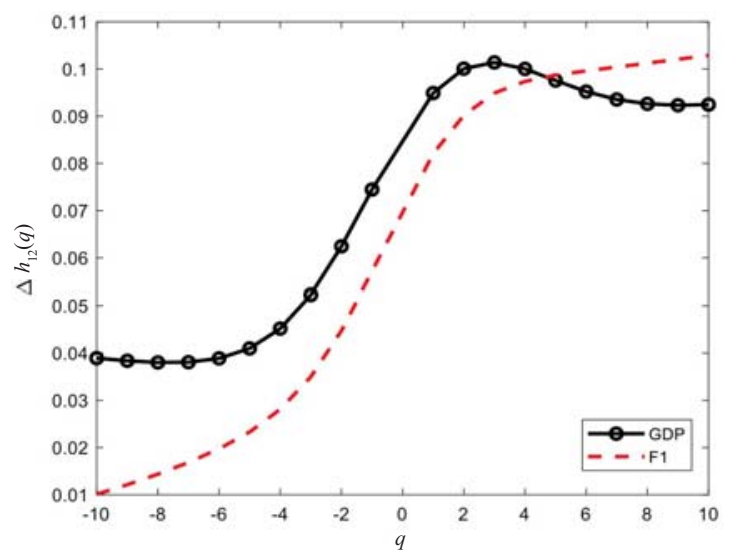

Figure $4 \Delta h_{12}(q)$ trends of GDP and F1 (REI)

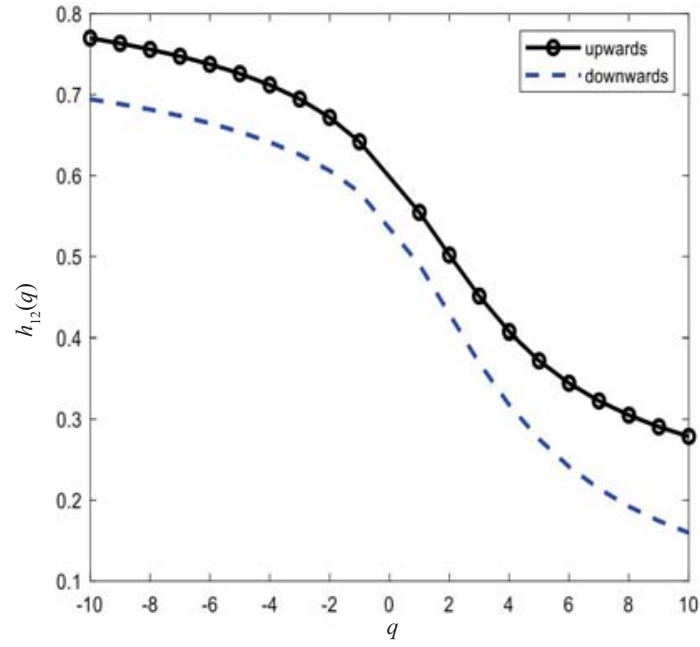

(a) GDP index

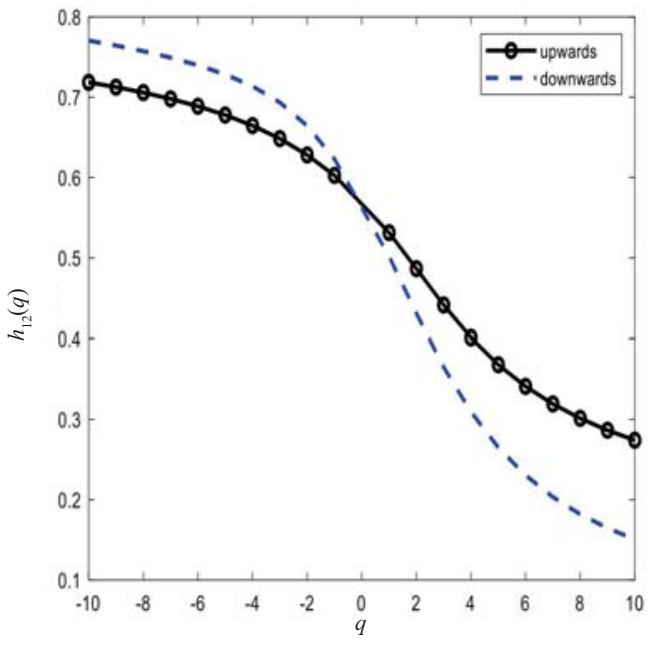

(b) CHS index

*Upwards means that the GDP and CHS growth rates are on the rise; downwards means that the GDP and CHS growth rates show a declining trend.

Figure 5 Relevance plots for $h_{12}^{+}(q)$ and $h_{12}^{-}(q)$

Regarding the CHS exponent, $h_{12}^{+}(q)$ is more than 0.5 for any $q$ value from -10 to $2, h_{12}^{+}(q)$ is less than 0.5 for any $q$ value from 2 to $10, h_{12}^{-}(q)$ is more than 0.5 for any $q$ value from -10 to 2 , and $h_{12}^{-}(q)$ is less than 0.5 for any $q$ value from 2 to 10 . They intersect when $q=0$. and the cross-relevance is less persistent when the CHS exponent shows an uptrend. In addition, the GDP exponent and CHS exponent both have multifractal asymmetric relevance relationships. Both $h_{12}^{+}(q)$ and $h_{12}^{-}(q)$ are decreasing functions of the value of $q$, but their change trends are not the same. 


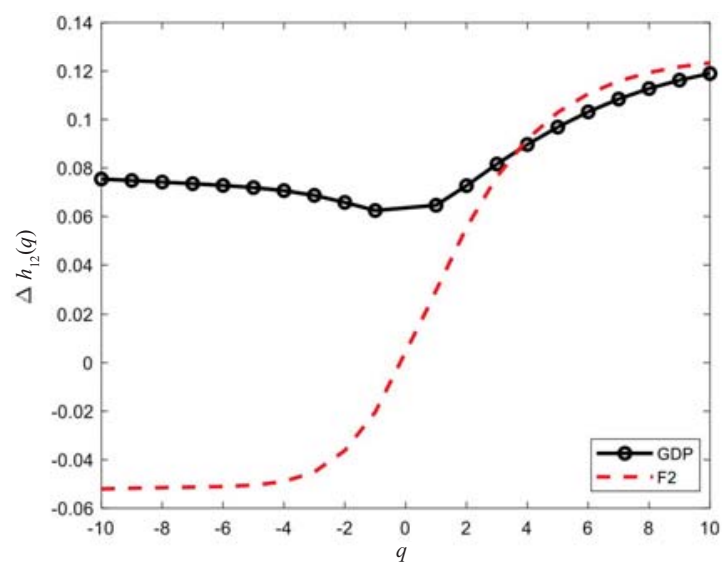

Figure $6 \Delta h_{12}(q)$ trends of GDP and F2 (CHS)

Based on Figure 6, both the GDP and CHS indexes increase the growth rate with an increase in the value of $q$, and they intersect when $q=4$. Additionally, from the trend direction, the $\Delta h_{12}(q)$ trends of GDP have a small fluctuation range, whereas the $\Delta h_{12}(q)$ trends of CHS have a large fluctuation range. It is also shown that $\Delta h_{12}(q)$ of GDP and CHS has asymmetric multifractal characteristics.

\subsection{Analysis Process of the DCCA Algorithm Based on a Time Delay}

The above sections (Subsection 4.1 and Subsection 4.2) use the DCCA algorithm and MFADCCA algorithm and show that there is a cross-correlation asymmetry relationship between GDP and REI and between GDP and CHS. However, they could not solve the issue of conduction direction between GDP and REI or between GDP and CHS.

First, $[1,12]$ is set as the value range of $\Delta T$, which represents the lag period from one month to one year. The $\Delta T$ increases by one unit, and the time delay is one month.

Figure 7 presents a trend chart of the change in $\Delta T$ with GDP index and REI index delays from 1 to 12 months. Regarding the GDP index, when $\Delta T$ increases from 1 to 5 and from 8 to $12, h_{\mathrm{GDP} \Delta T}(2)$ is larger than 0.5 . Additionally, when $\Delta T$ increases from 1 to $6, h_{\mathrm{GDP} \Delta T}(2)$ decreases. This means that in this period, with the time delay increase in GDP, the REI index and GDP growth rate become weak. However, as the time delay continues to increase, when $\Delta T$ increases from 8 to $10, h_{\mathrm{GDP} \Delta T}(2)$ is on the rise, which means that the REI index and GDP growth rate become strong. The REI index and GDP growth rate have unsteady fluctuations in cross-correlation persistence in the long term.

For the REI index, while $\Delta T$ increases from 1 to 5 and from 6 to $12, h_{\mathrm{REI} \Delta T}(2)$ is larger than 0.5. Additionally, when $\Delta T$ increases from 1 to $5, h_{\mathrm{REI} \Delta T}(2)$ decreases. This means that in this period, with the time delay increase in REI, the GDP index and REI growth rate become weak. However, as the time delay continues to increase, when $\Delta T$ increases from 6 to $10, h_{\mathrm{REI} \Delta T}(2)$ is on the rise, which means that the GDP index and REI growth rate become strong. The GDP index and REI growth rate have unsteady fluctuations in cross-correlation persistence in the long term.

In summary, when the GDP (REI) index in time delays can affect the REI (GDP) index, 
this effect diminishes quickly and increases in a short period of time, and there are unsteady fluctuations in cross-correlation persistence in the long term.

To measure the effects, define the difference between $h_{\mathrm{GDP} \Delta T}(2)$ and $h_{\mathrm{REI} \Delta T}(2)$ as the difference in cross-correlation, which is denoted as $\Delta h_{\mathrm{REI}-\mathrm{GDP}}^{\Delta T}(2)$,

$$
h_{\mathrm{REI}-\mathrm{GDP}}^{\Delta T}(2)=h_{\mathrm{REI} \Delta T}(2)-h_{\mathrm{GDP} \Delta T}(2) .
$$

Figure 8 presents the change in $\Delta h_{\mathrm{REI}-\mathrm{GDP}}^{\Delta T}(2)$ with time delays. $\Delta h_{\mathrm{REI}-\mathrm{GDP}}^{\Delta T}(2)$ is greater than 0 , which means that under the same time delays, the GDP growth rate has different effects on the REI index, and the REI index has stronger effects on the GDP growth rate.

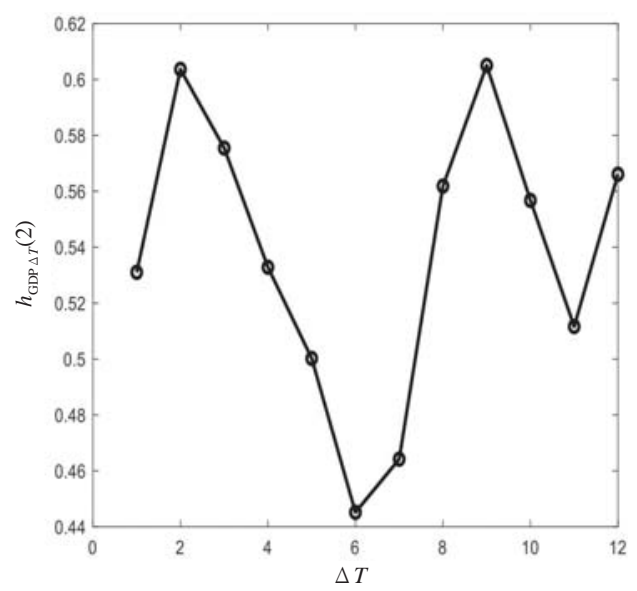

(a) GDP

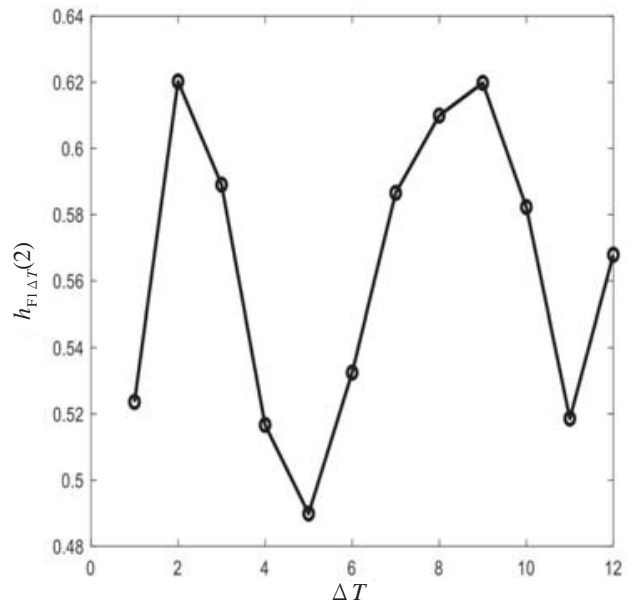

(b) F1 (REI)

Figure 7 Different time delay scaling exponents (GDP and REI)

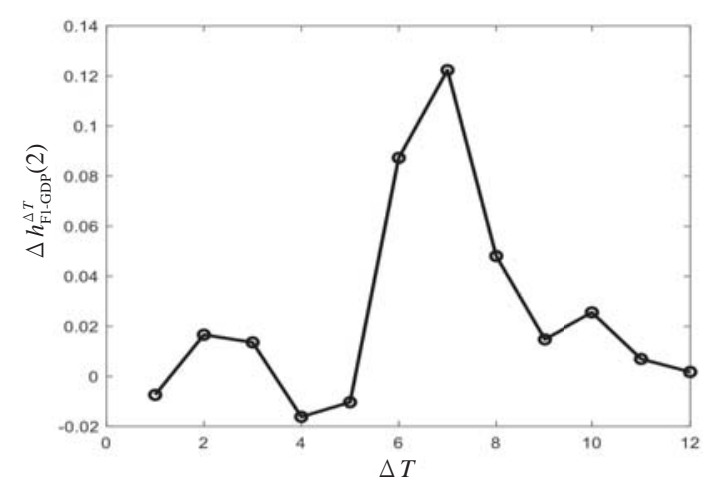

Figure 8 Difference in $\Delta h_{\mathrm{REI}-\mathrm{GDP}}^{\Delta T}(2)$ for different time delays

Figure 9 presents a trend chart of the change in $\Delta T$ with the GDP exponent and CHS exponent delays from 1 to 12 months. Regarding the GDP index, $h_{\mathrm{GDP} \triangle T}(2)$ is more than 0.5 , which means that while GDP has a delayed growth rate, its relevance is persistent. When $\Delta T$ increases from 2 to $6, h_{\mathrm{GDP} \triangle T}(2)$ decreases, which means that in this period, with the time delay increase in GDP, the CHS index and GDP growth rate become weak. However, as the 
time delay continues to increase, when $\Delta T$ increases from 6 to $9, h_{\mathrm{GDP} \Delta T}(2)$ is on the rise, which means that the CHS index and GDP growth rate become strong. The CHS index and GDP growth rate have unsteady fluctuations in cross-correlation persistence in the long term.

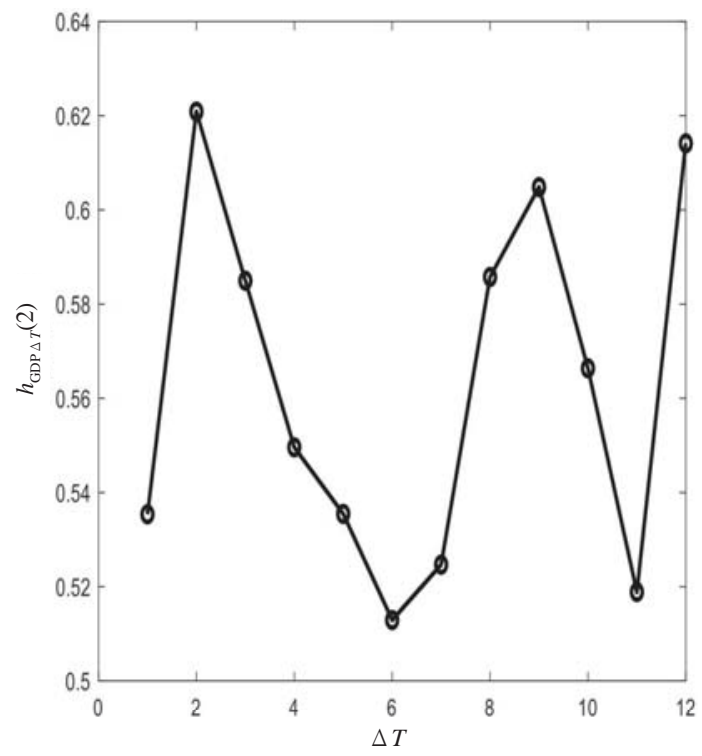

(a) GDP

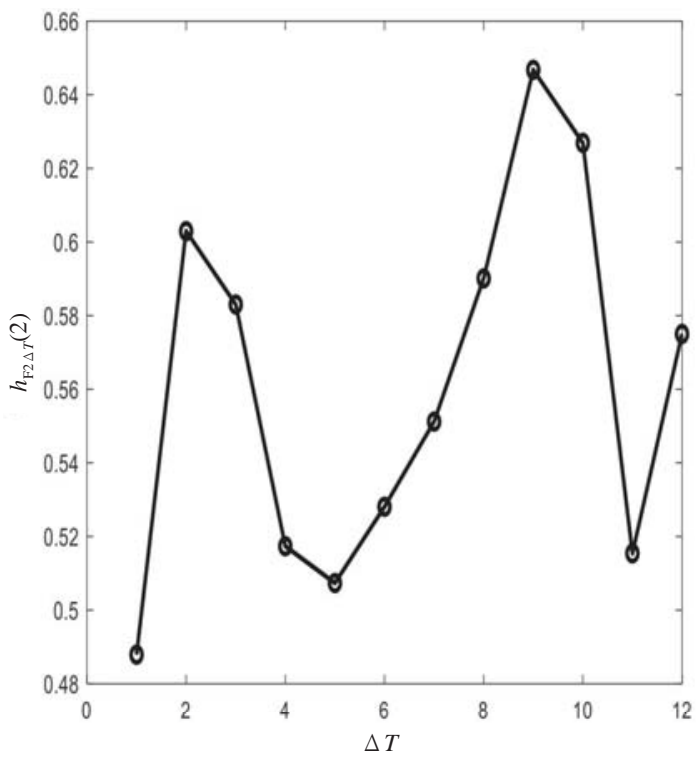

(b) F2 (CHS)

Figure 9 Different time delay scaling exponents (GDP and CHS)

Regarding the CHS index, when $\Delta T$ increases from 2 to $12, h_{\mathrm{CHS} \Delta T}(2)$ is more than 0.5 , which means that while CHS have a delayed growth rate, their relevance is persistent. Additionally, when $\Delta T$ increases from 2 to $5, h_{\mathrm{CHS} \Delta T}(2)$ decreases. With the time delay increase in REI, the GDP index and REI growth rate become weak in this period. Nevertheless, as the time delay continues to increase, when $\Delta T$ increases from 5 to $9, h_{\mathrm{CHS} \Delta T}(2)$ is on the rise, which means that the GDP index and REI growth rate become strong. The GDP index and REI growth rate have unsteady fluctuations in cross-correlation persistence in the long term.

In summary, when the GDP (CHS) index in time delays can affect the CHS (GDP) index, this effect diminishes quickly and increases in a short period of time, and there are unsteady fluctuations in cross-correlation persistence in the long term.

Similar to GDP and REI, to measure their effects, the difference between $h_{\mathrm{GDP} \Delta T}(2)$ and $h_{\mathrm{CHS} \Delta T}(2)$ is defined as the difference in the cross-correlation, which is denoted as $\Delta h_{\mathrm{REI}-\mathrm{CHS}}^{\Delta T}(2)$ :

$$
h_{\mathrm{CHS}-\mathrm{GDP}}^{\Delta T}(2)=h_{\mathrm{CHS} \Delta T}(2)-h_{\mathrm{GDP} \Delta T}(2) .
$$

Figure 10 presents the change in $\Delta h_{\mathrm{CHS}-\mathrm{GDP}}^{\Delta T}(2)$ with time delays. $\Delta h_{\mathrm{CHS}-\mathrm{GDP}}^{\Delta T}(2)$ is greater than 0 , which means that with the same time delays, the GDP growth rate has different effects on the CHS index, and the CHS index has stronger effects on the GDP growth rate. In contrast, $\Delta h_{\mathrm{CHS}-\mathrm{GDP}}^{\Delta T}(2)$ is less than 0 , which means that under the same time delays, the GDP growth rate has different effects on the CHS index, and the GDP index has stronger effects on the CHS growth rate. The reason is that GDP can affect CHS in the real estate market; that is, an increase in GDP affects CHS. 


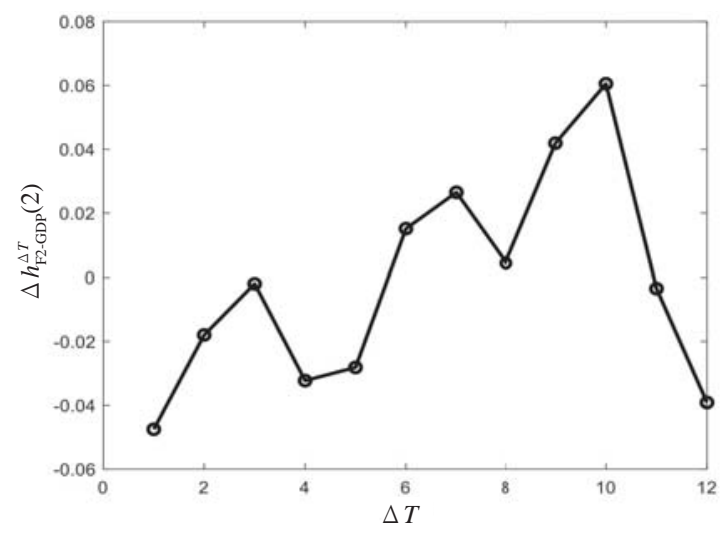

Figure 10 Difference in $\Delta h_{\mathrm{CHS}-\mathrm{GDP}}^{\Delta T}(2)$ for different time delays

\section{Robustness Test}

Section 3 presents the process of the DCCA algorithm, MF-ADCCA algorithm, and DCCA algorithm based on a time delay. To ensure the stability of the results of this paper, this section will perform a Robustness test.

According to Liu, et al. ${ }^{[10]}$, among the current DCCA algorithm references, some articles use the sliding time window algorithm to test the robustness of the results. This paper selected ten years of monthly data from 2010.02 to 2020.12 , and a total of 121 pieces of data were applied in this paper. Nevertheless, the sliding time window algorithm needs more than 700 data points. Thus, this method cannot be applied in this paper. In contrast, the Granger causality test can overcome this limitation and identify causality relationships between two indexes. Hence, this paper will perform a robustness test based on the Granger causality test. The robustness test results are shown in the tables below.

Based on Table 3, the $F$-statistics are 4.99329 and 2.67921, and they are both significant. The null hypothesis ("a decrease in real estate investment (DREI) does not Granger cause a decrease in GDP (DGDP)" or "a DGDP does not Granger cause a DREI") can be rejected. The results show a bilateral Granger causality link between GDP and REI. Meanwhile, the results of the DCCA algorithm have been verified as well.

Table 3 Pairwise Granger causality tests (GDP and REI)

\begin{tabular}{lllll}
\hline Null Hypothesis & Lags & Obs. & $F$-statistics & Prob. \\
\hline A DREI does not Granger cause a DGDP & 3 & 117 & 4.99329 & 0.0028 \\
A DGDP does not Granger cause a DREI & 3 & 117 & 2.67921 & 0.0505 \\
\hline
\end{tabular}

Based on Table 4, the $F$-statistics are 44.1616 and 20.6497, and they are both significant. The null hypothesis ("a DGDP does not Granger cause a decrease in commercial housing sales (DCHS)" or "a DCHS does not Granger cause a DGDP") can be rejected. The results show a bilateral Granger causality link between GDP and CHS. Meanwhile, the results of the DCCA algorithm have been verified as well. 
Table 4 Pairwise Granger causality tests (GDP and CHS)

\begin{tabular}{lllll}
\hline Null Hypothesis & Lags & Obs. & $F$-statistics & Prob. \\
\hline A DGDP does not Granger cause a DCHS & 1 & 119 & 44.1616 & 0.0000 \\
A DCHS does not Granger cause a DGDP & 1 & 119 & 20.6497 & 0.0000 \\
\hline
\end{tabular}

\section{Conclusion}

The result of the DCCA algorithm is that both REI and CHS have persistence with GDP. This also means that while GDP rises in the current period, in the next period, it will decrease if there are no external factors.

The results of the MF-ADCCA algorithm are as follows: 1) While the GDP exponent shows a declining trend, its anti-persistence is stronger than it is when the GDP exponent is on the rise. While the REI and CHS growth rates are on the rise, their cross-correlation is more persistent. 2) The effects on the REI (CHS) growth rate rely on the magnitude of the fluctuations based on the GDP exponent. While the GDP exponent is on the rise, the cross-correlation of large fluctuations is less persistent, whereas small fluctuations are more persistent. 3) The influence of the REI (CHS) exponent on the GDP growth rate does not depend on each other, and their relevance is less persistent, while the REI (CHS) exponent presents a rising trend. 4) The GDP exponent, REI exponent and CHS exponent have asymmetric multifractal characteristics.

The results of the DCCA algorithm based on a time delay are as follows: 1) The GDP (CHS, REI) index in time delays can affect the CHS and REI (GDP) indexes. This effect diminishes in a short period of time, and there are unsteady fluctuations in cross-correlation persistence in the long term. 2) The GDP growth rate has different effects on the REI and CHS indexes; otherwise, an increase in GDP can positively affect CHS and REI in the real estate market. Decreasing GDP can negatively affect CHS and REI in the real estate market.

\subsection{Discussion}

The above results indicate that high-quality economic development cannot over-rely on real estate industry development. In contrast, high-quality economic development can increase real estate industry development in the long term. Based on the above results, the real estate industry should set up healthy goals for long-term development. It is better for high-quality economic development to lead to real estate industry development. Regardless, the economy of China should develop from high speed to high quality.

In current research, the relationship between the real estate industry and the economy is still mainly viewed from two perspectives. One view posits that the real estate industry increases economic development, while the other view posits that the real estate industry does not increase economic development. These results are based on different perspectives, research methods and variables. For example, based on a real estate price variable, Guo \& $\mathrm{Li}^{[2]}$ found that the real estate industry has a direct inhibitory effect and indirect promoting effect. Using the panel vector autoregressive (PVAR) model, Ren \& Chen ${ }^{[24]}$ stated that there is an inverted U-shaped relationship between real estate and GDP. Zhu ${ }^{[25]}$ obtained the same result as Ren Wei but with different research methods. Using the dynamic computable general equilibrium (DCGE) model, Yuan \& Feng ${ }^{[26]}$ proved that the real estate industry can bring economic 
growth, but it will squeeze the development space of other industries, such as light industry. Based on the situation of the economic development period (high-speed period, smooth period, ripening period), real estate prices can increase rapidly in the high-speed period ${ }^{[27]}$.

Based on the above studies, the healthy development of the real estate industry is important for China's high-quality economic development. In future research, it will be necessary for more scholars to take other perspectives or to adopt other research methods to conduct research in this field.

\subsection{Recommendations}

Based on the results of this paper, several recommendations are listed below:

a) Reasonably plan REI in the market. It is necessary to improve the proportion of infrastructure investment and to optimize the urban industry layout. Decreasing REI can provide a better economic environment. As shown in the results of this paper, REI relies on the magnitude of fluctuations based on GDP.

b) Control the growth speed of real estate prices. Higher real estate prices will affect CHS because a fast speed of increase will cause unreasonable economic development. The effect of CHS on the GDP growth rate does not depend on each other, and their relevance is less persistent. However, in the DCCA algorithm based on a time delay, CHS have a stronger effect on the GDP growth rate.

c) Establish differentiated regulation of different regions. Since GDP, REI, and CHS have asymmetric multifractal characteristics, different regions of China can use specific situations to ensure the quality of economic development.

Acknowledgements Thankful for the comments and suggestions that given by the reviewers. They have improved the content of this paper and pointed out research directions of particular interest.

\section{References}

[1] Ren B P. Quality of economic growth: Expansion of theoretical of economic growth. Economic Trends, 2013(11): 45-51.

[2] Guo W W, Li J Q. Does housing bubble inhibit high-quality economic growth? — Based on the experience analysis of 13 economic circles in China. China Soft Schience, 2019(8): 77-91.

[3] China Development Research Foundation "Primus Macro Forum" Medium - and Long-Term Development Research Group. Liu S J, Wang Z H, Cai J T, et al. 2035: The potential, structure and path of China's Economic Growth. Management World, 2018, 34(7): 1-12+183, doi: 10.19744/j.cnki.11-1235/f.2018.07.002.

[4] Liu C, Li J Y, Wang C, et al. Relations of real estate development, economic growth driving factors, external environment and economic growth effect in China: Based on time series data during 2000-2016. Management Review, 2018(8): 30-35.

[5] Zhang Y Q, Wang S Y. Trade potential of China's export to ASEAN: The gravity model using new economic mass proxies. Journal of Systems Science and Information, 2015, 3(5): 411-420.

[6] Bhowmik R, Chao W U, Roy K J, et al. A study on the volatility of the Bangladesh stock market — Based on GARCH type models. Journal of Systems Science and Information, 2017, 5(5): 193-215.

[7] Wolfgang B I. Recent trends in real estate research: A comparison of recent working papers and publications using machine learning algorithms. Journal of Business Economics, 2020(90): 963-974.

[8] Tommaso G, Keith P, Tianyu W. Estimation of bubble dynamics in the Chinese real estate market: A state space model. International Economics and Economic Policy, 2018(15): 483-499. 
[9] Balemi N, Fäss R, Weigand A. COVID-19's impact on real estate markets: Review and outlook. Financial Markets and Portfolio Management, 2021, 35: 495-513.

[10] Liu C, Zheng Y, Liu C Q, et al. Is the stock market a barometer of the economy? Based on 2005-2017 years' CSI 300 index and PMI data. Systems Engineering — Theory \& Practice, 2020, 40(1): 55-68.

[11] Zhou H B, Fan Z G, Wang C, et al. Study on the quality effect of economic growth of real estate investment in China under the new development concept. Review of Investment Studies, 2020, 39(5): 37-51.

[12] Jing G, Wang L G. Research on the effect of real estate investment on China's economic growth. Review of Investment Studies, 2019, 38(4): 80-92.

[13] Zhang J X. Study on the risk measurement of Beijing real estate market — Based on the empirical analysis of Markov zone system transfer model. The World of Survey and Research, 2021(3): 39-48.

[14] Fan X Y, Wang Y H, Zhang S D. Research of inhibition effect of real estate prices on innovation based on the aspects of transfer of capital from the virtual to the real. Journal of Applied Statistics and Management, 2021, doi: 10.13860/j.cnki.sltj.20210305-028.

[15] Wu J X, Lu C. The impact of real estate investment on the economy. Construction Economics, 2020, 41(S2): 249-250.

[16] Zhang X Y, Teng Y Y. Is real estate growth conducive to real economy growth? — Research on the mediating effect of corporate profits. Shandong Social Sciences, 2019(11): 291-296.

[17] Cui G L, Gao T H. Real estate investment, resident consumption and urban economic growth. Systems Engineering - Theory \& Prantice, 2020, 40(7): 1655-1670.

[18] Cui J X, Zou H W. Connectedness among economic policy uncertainties: Evidence from the time and frequency domain perspectives. Journal of Systems Science and Information, 2020, 8(5): 401-433.

[19] Li G B, Wang J. The impact of housing prices on the quality of economic growth in China: An empirical study based on panel data of 286 cities at and above the prefectural level. Prices Monthly, 2018(5): 89-98.

[20] Chen B K, Huang S A, Ouyang D. Can growing housing price drive economic growth? China Economic Quarterly, 2018(3): 126-131.

[21] Yang J J. The micro effect of real estate price fluctuation on macroeconomic fluctuation. Economic Research Journal, 2012(S1): 117-127.

[22] Ouyang Z G, He F M, Xue L. Economic policy uncertainty, dual-wheel drive and economic growth. Systems Engineering - Theory \& Practice, 2019, 39(4): 986-1000.

[23] Hasan R, Salim M M. Power law cross-correlations between price change and volume change of Indian stocks. Physica A: Statistical Mechanics and Its Applications, 2017, 473: 620-631.

[24] Ren W, Chen L W. Urbanization, urban-rural income gap and housing price fluctuation. Price Monthly, 2019(6): 14-21.

[25] Zhu L N. Mechanism and path analysis of housing price's impact on economic growth. Statistics and Decision, 2016(20): 140-143.

[26] Yuan P F, Feng L. Economic growth, income distribution and wealth differentiation: A study on the effect of real estate price rise based on DCGE model. Economic Research, 2014(9): 77-90.

[27] Zhong W, Wei W. Economic growth, exchange rate and real estate: International comparisons. International Journal of Finance, 2014(6): 3-9. 